\title{
Disorder-induced domain wall velocity shift at high fields in perpendicularly magnetized thin films
}

\author{
Michele Voto, ${ }^{*}$ Luis Lopez-Diaz, Luis Torres, and Simone Moretti \\ Departamento de Física Aplicada, Universidad de Salamanca, Plaza de la Merced s/n, 37008 Salamanca, Spain
}

(Received 2 August 2016; revised manuscript received 14 October 2016; published 23 November 2016)

\begin{abstract}
Domain wall dynamics in a perpendicularly magnetized system is studied by means of micromagnetic simulations in which disorder is introduced as a dispersion of both the easy-axis orientation and the anisotropy constant over regions reproducing a granular structure of the material. High field dynamics show a linear velocity-field relationship and an additional grain size dependent velocity shift, weakly dependent on both applied field and intrinsic Gilbert's damping parameter. We find the origin of this velocity shift in the nonhomogeneous in-plane effective field generated by the tilting of anisotropy easy axis introduced by disorder. We show that a one-dimensional analytical approach cannot predict the observed velocities and we augment it with the additional dissipation of energy arising from internal domain wall dynamics triggered by disorder. This way we prove that the main cause of higher velocity is the ability of the domain wall to irradiate energy into the domains, acquired with a precise feature of disorder.
\end{abstract}

DOI: 10.1103/PhysRevB.94.174438

\section{INTRODUCTION}

Domain wall (DW) dynamics in ultrathin films with high perpendicular magnetocrystalline anisotropy (PMA) has received a lot of attention over the last years [1-3] since these structures present several advantages over in-plane magnetized materials towards their potential use in spintronic devices, such as narrower domain walls and better scalability. The success of domain wall based devices [3-5] relies entirely on the ability to control their position and their motion reliably. The main features of field-driven domain wall dynamics in ferromagnetic strips are well captured by the one-dimensional model of Walker and Slonczewski [6,7]. This model predicts a velocity field dependence characterized by two main regimes: the linear steady regime below the Walker field $H_{a}<H_{W}$, where the DW moves rigidly and its velocity dependence on driving field $H_{a}$, the mobility, is inversely proportional to Gilbert's damping parameter $\alpha$, and the linear precessional regime [8,9], where the mobility is proportional to $\left(\alpha+\alpha^{-1}\right)^{-1}$. These two linear regimes are separated by an intermediate regime after Walker field, where velocity shows a nonlinear dependence on the driving field [7].

On the other hand, it has recently been shown that features like dynamic DW deformations and variations in its internal structure, which are beyond the one-dimensional model, significantly affect its velocity [10-13]. In particular, recent works have focused their attention on the role played by motion and annihilation of vertical Bloch lines (VBL) inside the DW and how these processes have a strong impact on the DW velocity [11]. Such features cannot be taken care of in the one-dimensional model and, consequently, micromagnetic simulations have become an essential tool for an interpretation of these complexities, allowing for a better understanding of experimental data.

Moreover, it is well known that material inhomogeneities and disorder also affect DW dynamics because they act as pinning centers. In particular, a finite minimum field, called depinning field $\left(H_{\mathrm{dep}}\right)$, is required to propagate the DW in every

*michele.voto@usal.es sample. For fields smaller than $H_{\text {dep }}$ the so-called creep regime is observed $[8,14]$, where DW motion takes place via thermally activated localized depinning events. For fields higher than $H_{\text {dep }}$ the wall accelerates up to a viscous flow regime [15]. Due to disorder, then, the transition between the steady and precessional regimes predicted by the one-dimensional model is hardly observed experimentally. This is particularly the case in PMA films, where the Walker breakdown field is typically much smaller than the depinning field. In any case, it is clear that micromagnetic simulations are needed to properly investigate the effect of disorder in DW motion. They were used in $[16,17]$ to study the motion of vortex domain walls in soft in-plane magnetized nanostrips and it was shown [16] that disorder introduces a new means of dissipating the energy in the system via spin wave emission during DW propagation, which was characterized by an extrinsic contribution to the Gilbert damping parameter. Extrinsic contributions to damping during DW propagation are also emphasized in [18], where it is shown that the experimental value of damping extracted from FMR measurements is considerably smaller than the one extracted from DW mobility in permalloy strips. On the other hand, in a recent work [19], we used a micromagnetic model with an irregular grain structure to show that a certain degree of inhomogeneity is required to account for the DW velocities measured experimentally in thin films with PMA in the typical plateau region obtained in the viscous regime at intermediate fields [20,21].

At very high fields, however, when the force exerted by the field on the wall is much larger than the pinning force due to disorder $\left(H_{a} \gg H_{\text {dep }}\right)$, the effect of nonuniformities becomes negligible and, therefore, DW deformations should be minimal and good agreement with the linear precessional regime predicted by the one-dimensional model is expected $[7,8]$. In the present work we focus our attention on this high-field regime. Using the micromagnetic model presented in [19], which has been proven to adequately describe experimental data, we observe that the introduction of material disorder preserves a linear field-velocity dependence at high fields, but a fixed contribution to DW velocity appears, yielding a velocity shift that depends on the chosen grain size. We verify that this contribution to DW velocity is solely due to 

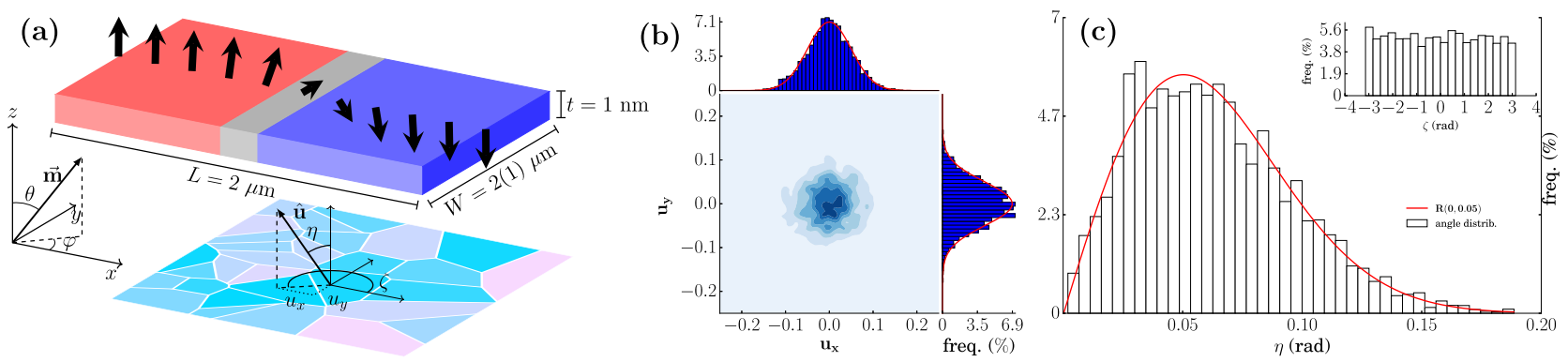

FIG. 1. (a) Schematic of the system under study: Bloch domain wall separating up and down domains. The external field is applied out-of-plane, favoring the expansion of the red domain. A typical Voronoi tessellation for a grain distribution is shown on the $x$ - $y$ plane together with the polar $(\eta)$ and azimuthal ( $\zeta$, inset) angles of a tilted anisotropy easy-axis $\hat{u}$. (b) Distribution of the in-plane components of $\hat{u}$ for a sample with grain size $d=30 \mathrm{~nm}$. A Gaussian distribution of standard deviation $\sigma=0.05$ and mean 0 is used to perturb the $x$ and $y$ components at each grain. (c) Corresponding distribution of the polar $(\eta)$ and azimuthal $(\zeta)$ angles of the easy axis for the same sample.

the dispersion in the anisotropy easy-axis $\hat{u}$ tilting, which generates a local in-plane field that varies from grain to grain. The effect of this local in-plane field is twofold: on one hand, a local net contribution to the out-of-plane torque on the DW spins arises from a tilted easy axis and can be accounted for in the one-dimensional model analysis. On the other, the velocity of the VBLs propagating along the DW is sensitive to in-plane fields, which gives rise to annihilation events and complex dynamics of the DW that translate into an augmented spin wave radiation from the DW.

The manuscript is organized as follows. The simulated system and the methods are described in Sec. II. In Sec. III we present the results of simulations for different grain parameters, and we identify the feature of the model that causes the velocity shift. Section IV is dedicated to enhance the analytical model considering the different DW energy dissipation mechanisms in a homogeneous film and in presence of disorder. We compare the mechanisms both qualitatively and quantitatively giving a correction to the analytical model prediction for DW velocity. In Sec. V we summarize the main results of the paper.

\section{METHODS}

The system under study is represented schematically in Fig. 1(a). The values of the material parameters have been chosen to mimic those of annealed $\mathrm{Ta} / \mathrm{Co}_{20} \mathrm{Fe}_{60} \mathrm{~B}_{20} / \mathrm{MgO}$ thin films studied and characterized in [20,22]: exchange constant $A=23 \times 10^{-12} \mathrm{~J} / \mathrm{m}$, uniaxial anisotropy constant $k_{u}=8.34 \times 10^{5} \mathrm{~J} / \mathrm{m}^{3}$, easy-axis $\hat{u}=\hat{z}$, and saturation magnetization $M_{s}=8.83 \times 10^{5} \mathrm{~A} / \mathrm{m}$.

The computational region is a rectangle $L \times W$ with $L=$ $W=2 \mu \mathrm{m}$ and the film thickness is $1 \mathrm{~nm}$. No relevant effect of Dzyaloshinskii-Moriya interaction (DMI) is observed in this system $[23,24]$, hence we do not include it in our simulations. The effect of disorder in the material is modeled via a Voronoi tessellation of the film in polygonal regions of average diameter $d$ that reproduces the grain structure appearing upon annealing in the material. A different value of anisotropy constant $k_{u, i}$ and easy-axis orientation $\hat{u}_{i}$ is assigned to each grain, following a Gaussian distribution around their nominal value with standard deviation $\sigma=5 \%$. To investigate DW dynamics, we integrate the Landau-Lifshitz-Gilbert (LLG) equation

$$
\frac{d \mathbf{M}}{d t}=-\gamma \mathbf{M} \times \mathbf{H}_{\mathrm{eff}}+\frac{\alpha}{M_{s}} \mathbf{M} \times \frac{d \mathrm{M}}{d t},
$$

solved numerically via Runge-Kutta 4 (RK4) algorithm with a time-step $h=400 \mathrm{fs}$, using a custom finite difference micromagnetic solver tested against popular open source solvers (Mumax3 [25], OOMMF [26]) and dividing the computational region in $4 \times 4 \times 1 \mathrm{~nm}^{3}$ cells.

The initial magnetization for our simulations is a symmetric up-down configuration with two domains separated by a Bloch wall in the middle. After relaxing our system to equilibrium obtaining a magnetic configuration $\mathbf{M}_{0}$, an external field is applied along the positive $\hat{\mathbf{z}}$ direction inducing the motion of the DW towards the right. The dynamics is simulated until one of two stopping criteria is met: $t \geqslant 50 \mathrm{~ns}$ or $\left\langle m_{z}\right\rangle \geqslant 0.8$. DW velocity is computed as $\frac{L}{2} \Delta\left\langle m_{z}\right\rangle / \Delta t$, where $\Delta\left\langle m_{z}\right\rangle$ is the variation in the average $z$ component of the magnetization during the time window $\Delta t$. For each value of the applied field the initial configuration is set to $\mathbf{M}_{0}$. We generate six different samples for different grain sizes $d=5,10, \ldots, 30 \mathrm{~nm}$ and a sample with no disorder. For each of the samples we measure DW velocity for 12 different applied fields between 20 and $360 \mathrm{mT}$ and six different values of Gilbert's damping parameter $\alpha=0.005,0.01,0.015,0.02,0.03,0.05$, where $\alpha=$ 0.015 is the experimentally measured value [27].

In a subsequent part of our study we reduce the width of our magnetic sample to $W=1 \mu \mathrm{m}$ and the lateral size of the cells to $3.90625 \mathrm{~nm}$ to have a power of two number of cells for faster simulation. We replicate the DW velocity measurements on these new samples, using a time-step $h=200 \mathrm{fs}$ and we save magnetization configuration every 20 ps together with the distribution of dissipated energy density computed as

$$
\Delta \mathcal{E}\left(\mathbf{r}, T_{k}\right)=\mu_{0} M_{s} \sum_{i=1}^{100} \Delta \mathbf{m}\left(\mathbf{r}, h_{i, k-1}\right) \cdot \mathbf{H}_{\mathrm{eff}}\left(\mathbf{r}, h_{i, k-1}\right),
$$

where $\Delta \mathbf{m}\left(\mathbf{r}, h_{i, k}\right)$ is the magnetization increment at time $h_{i, k}=i h+T_{k}$ provided by the numeric solver and $\mathbf{H}_{\mathrm{eff}}\left(\mathbf{r}, h_{i, k}\right)$ is the corresponding effective field. Dissipated energy distribution allows us to observe the areas that are more affected by energy dissipation and any relevant localized event of energy dissipation that takes place during the dynamics. 

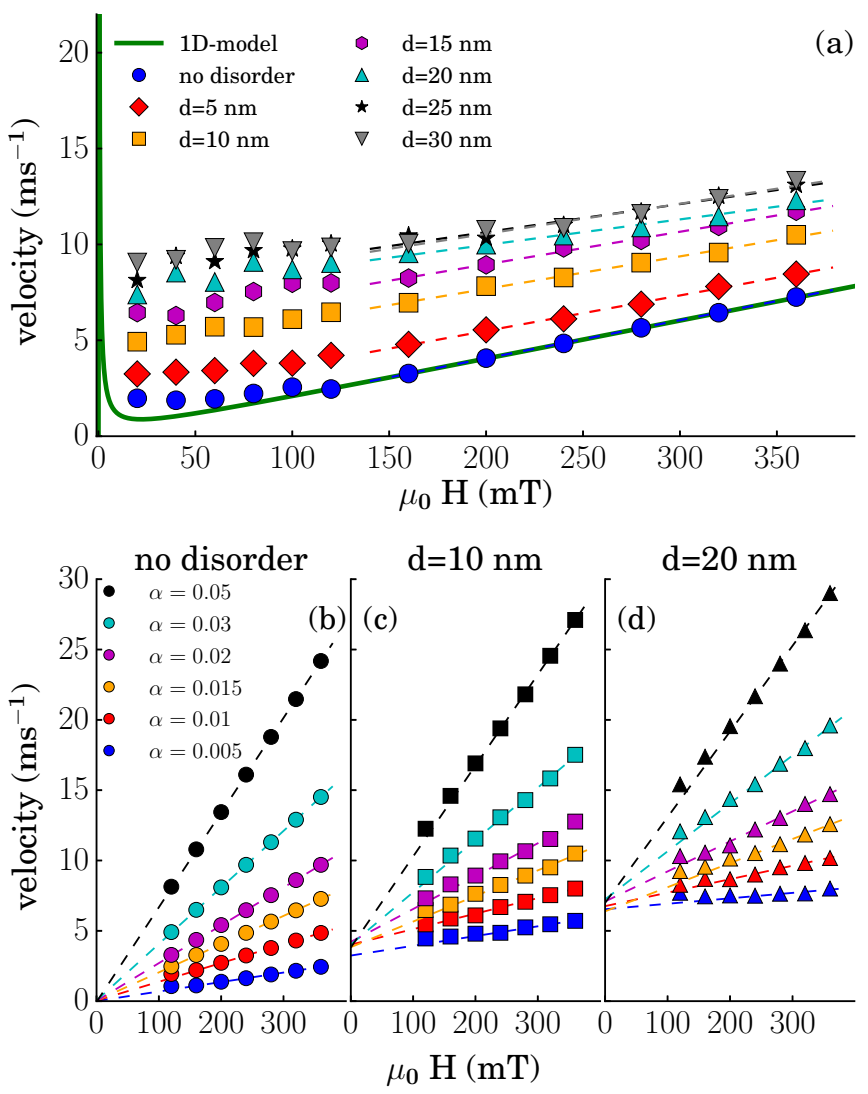

FIG. 2. (a) DW velocity as function of applied field from micromagnetic simulations with Gilbert's damping $\alpha=0.015$ and various grain diameters, together with simulations with no disorder and 1D model analytical calculations (green line). (b)-(d) DW velocity at high field for various values of $\alpha$ and a fixed grain size, showing the shift in the zero field intercept with introduction of disorder. Dashed lines are linear fittings to the 1D linear precessional regime.

\section{RESULTS AND ANALYTICAL APPROACH}

DW velocity measurements from micromagnetic simulations are shown in Fig. 2(a) for samples of width $W=2 \mu \mathrm{m}$ with various grain sizes and $\alpha=0.015$, together with the one-dimensional (1D) model calculations. As can be observed, for applied fields greater than Walker field $H_{W} \sim 1 \mathrm{mT}$, $1 \mathrm{D}$ model predicts a nonlinear precessional periodic regime for which the following expression for the average velocity $\langle v\rangle=T^{-1} \int_{T} v d t$ can be obtained [9]:

$$
\begin{aligned}
v(t) & =\frac{\gamma_{0} \Delta}{1+\alpha^{2}}\left(\alpha H_{a}+H_{K} \frac{\sin 2 \phi}{2}\right), \\
\langle v\rangle & =\frac{\alpha \gamma_{0} \Delta}{1+\alpha^{2}} H_{a}+\frac{\gamma_{0} \Delta}{\alpha\left(1+\alpha^{2}\right)} \underbrace{\left[H_{a}-\sqrt{H_{a}^{2}-H_{W}^{2}}\right]}_{\rightarrow 0 \text { for } H_{a} \gg H_{W}} .
\end{aligned}
$$

Here $H_{K}=2 K / \mu_{0} M_{s}$ is the shape anisotropy field acting on the DW, $H_{W}=\alpha H_{K} / 2$ is the Walker's field, $\gamma_{0}=2.21 \times$ $10^{5}(\mathrm{~A} / \mathrm{m})^{-1} \mathrm{~s}^{-1}$ is the (positive) gyromagnetic ratio, and $\phi$ is the angle describing the in-plane orientation of the DW spins. A full derivation of the equations can be found in the Appendix. On the other hand, a plateau for the velocity is found in simulations of wide wires [7,20]. As discussed in our previous work [19], the existence of this velocity plateau and deviation from the one-dimensional model has its origin in the non-one-dimensional character of the system and the consequent nonuniform magnetization along the DW length, generated by nonuniformities in the stray field along the DW that yields a saturation velocity [7]. At higher fields, this stray field inhomogeneity becomes less and less important and a good agreement with the 1D model is found for the sample with no disorder (blue circles), where the spins inside the DW precess coherently. This means that for $H_{a} \gg H_{W}$, the DW in an ideally perfect sample behaves in a quasi1D fashion, precessing uniformly and showing the linear dependence of velocity on field predicted by Eq. (3), $\langle v\rangle=$ $\gamma_{0} \Delta H_{a}\left(\alpha+\alpha^{-1}\right)^{-1}$. The introduction of disorder, however, causes a general shift in the velocity both in the plateau and in the precessional linear regime. Velocity is increased by an amount that is dependent on grain size and saturates at around 25-30 nm but it is weakly dependent on the applied field.

Variation of $\alpha$ parameter affects the dynamics of our system changing the slope of the linear field-velocity relationship, as prescribed by Eq. (3). Figures 2(b)-2(d) presents DW velocity measurements for the sample without disorder (b) and samples with grain size $d=10 \mathrm{~nm}$ (c) and $20 \mathrm{~nm}(\mathrm{~d})$, where the velocity values at lower fields have been omitted to make clearly visible the zero field intercept of the linear fitting. It appears that the introduction of disorder gives rise to an additional term of the velocity in the precessional linear regime, changing the velocity expression in this regime to

$$
\langle v\rangle \simeq \frac{\alpha \gamma_{0} \Delta}{1+\alpha^{2}} H_{a}+v_{0}(d)
$$

It is clear that, in the high field linear regime investigated here, a characterization of the effect of disorder as a variation in the damping parameter as done in [16-18] does not apply, since disorder does not affect the DW mobility, but it introduces a shift in the velocity. Rather, one would tend to think, at least phenomenologically, that the effect of disorder is decoupled from the contribution due to damping.

To get further insight into the effect of disorder on DW dynamics, we consider separately the two different ways in which disorder is introduced in our model, namely the dispersion in $k_{u}$ and $\hat{u}$.

Figure 3(a) shows the simulations for a sample with grains of $10 \mathrm{~nm}$ diameter together with the perfect film and simulations of that very sample where the only parameter varying in space is either $k_{u}$ (spades symbol $\mathbf{A}$ ) or $\hat{u}$ (clubs symbol \$). As can be seen, the influence on DW velocity of the tilting of $\hat{u}$ is striking, while $k_{u}$ distribution does not seem to play a role. Although variation of both parameters is relevant when studying DW depinning and low field dynamics [17-19,28], at high fields the local variation of $k_{u}$ has negligible effects. Our interpretation for this is the fact that the value of $k_{u}$ influences the width and linear energy density of a DW, hence playing a bigger role in the statics. A deviation of $\hat{u}$ from the normal to plane, on the other hand, introduces directly an inplane transverse anisotropy. The effect of in-plane anisotropy on the DW dynamics can be investigated by including it in the 1D model of Slonczewski [7]. 

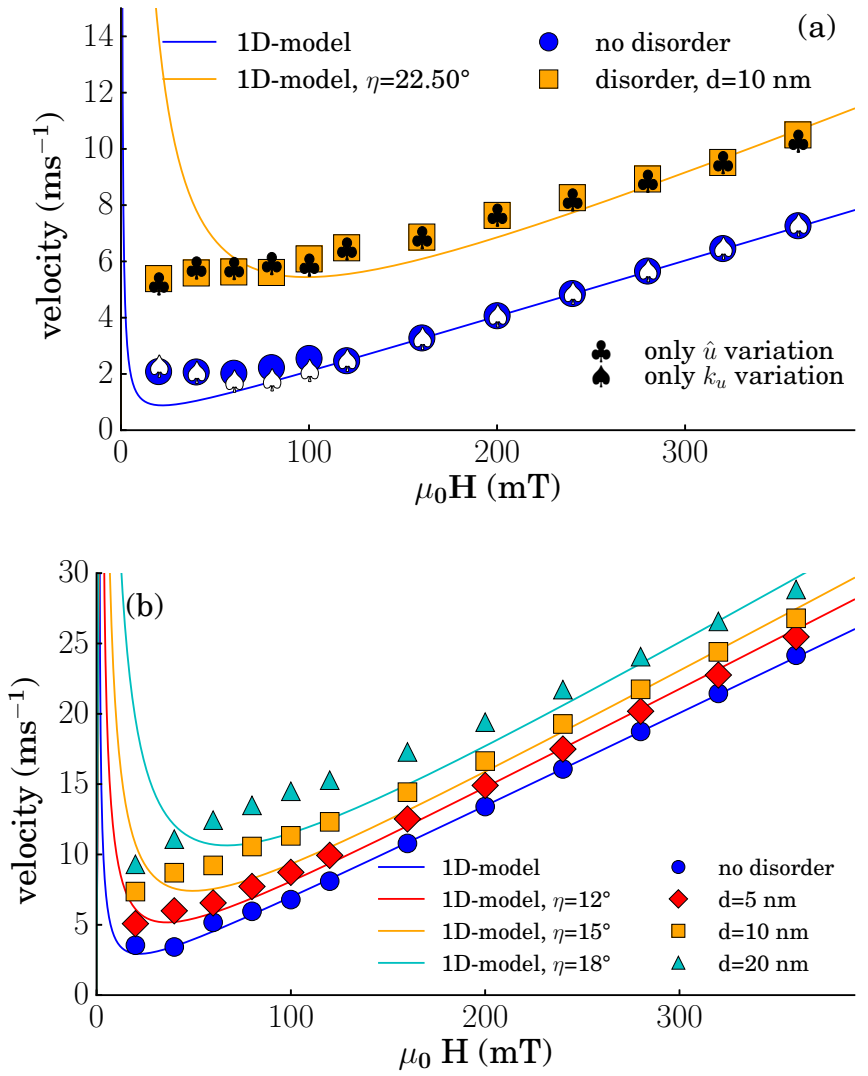

FIG. 3. (a) DW velocity versus field from micromagnetic simulations with Gilbert's damping $\alpha=0.015$ of a perfect sample (blue circles) and a sample with disorder and grain size $d=10 \mathrm{~nm}$; continuous lines are 1D model velocity calculations with a specified tilting $\eta$ of easy axis; spades $(\boldsymbol{\Lambda})$ symbols show DW velocity for simulations where easy axis has been kept uniform $\hat{u}=\hat{z}$; clubs (\$) symbols show DW velocity for simulations where anisotropy constant $k_{u}$ has been kept uniform. (b) DW velocity versus field from simulations with $\alpha=0.05$ and various grain size. Continuous lines are 1D model velocity calculations for various $\eta$.

The equations of motion for an up-down domain wall in case of a tilted anisotropy axis $\hat{u}=\left(u_{x}, u_{y}, u_{z}\right)=$ $(\sin \eta \cos \zeta, \sin \eta \sin \zeta, \cos \eta)$ become

$$
\begin{aligned}
v=\dot{q} & =\frac{\gamma_{0} \Delta}{1+\alpha^{2}}\left(\alpha H_{a}+\frac{H_{K}}{2} \sin 2 \phi-H_{K}^{\prime \prime} \cos 2 \phi\right) \\
& =\frac{\gamma_{0} \Delta H_{W}}{1+\alpha^{2}}\left(\alpha h_{1}+\frac{1}{\alpha}\left(\sin 2 \phi-h_{2} \cos 2 \phi\right)\right), \\
\dot{\phi} & =\frac{\gamma_{0}}{1+\alpha^{2}}\left(H_{a}-\frac{\alpha H_{K}}{2} \sin 2 \phi+\alpha H_{K}^{\prime \prime} \cos 2 \phi\right) \\
& =\frac{\gamma_{0} H_{W}}{1+\alpha^{2}}\left(h_{1}-\sin 2 \phi+h_{2} \cos 2 \phi\right),
\end{aligned}
$$

where $q$ is the $1 \mathrm{D}$ model DW position along the strip and

$$
\begin{aligned}
K & =\frac{1}{2} \mu_{0} M_{s}^{2}\left(N_{y}-N_{x}\right)-k_{u}\left(u_{y}^{2}-u_{x}^{2}\right), \\
H_{K}^{\prime \prime} & =\frac{2 k_{u} u_{x} u_{y}}{\mu_{0} M s}, \quad h_{1}=\frac{H_{a}}{H_{W}}, \quad h_{2}=\frac{2 H_{K}^{\prime \prime}}{H_{K}},
\end{aligned}
$$

$N_{x}$ and $N_{y}$ being the DW demagnetizing factors along $x$ and $y$. A complete discussion and derivation of the equations is given in the Appendix.

For fields above Walker breakdown, $h_{1} \geqslant \sqrt{1+h_{2}^{2}}$, we find again a periodic motion and an average DW velocity

$$
\langle v\rangle=\frac{\gamma_{0} \Delta H_{W}}{1+\alpha^{2}}\left[\alpha h_{1}+\frac{1}{\alpha}\left(h_{1}-\sqrt{h_{1}^{2}-1-h_{2}^{2}}\right)\right] .
$$

Note that (6) is exactly equivalent to (3) in the case $\hat{u}=\hat{z}$ and it describes DW velocity in the precessional regime for a DW propagating in one-dimensional system with a uniformly tilted anisotropy axis.

There are several differences between the 1D system described by (6) and the system studied in micromagnetic simulations: the easy axis does not have a uniform direction but is randomly tilted from the out-of-plane direction following a normal distribution of mean $\hat{z}$ and standard deviation $\sigma=5 \%$. This gives a Rayleigh distribution for the polar angle $\eta$ of $\hat{u}$ and a uniform distribution in $[0,2 \pi)$ for its azimuthal angle $\zeta$ as shown in Fig. 1(c). A fixed polar and azimuthal angle are required for the $1 \mathrm{D}$ model prediction. Since the choice of azimuthal angle $\zeta$ highly affects the dynamics and all orientations have the same probability [see inset of Fig. 1(c)], we choose only the polar angle $\eta$ and calculate the velocity as a mean of the values obtained varying $\zeta$ over all the interval $[0,2 \pi)$. 1D model calculations compared to simulations are shown in Figs. 3(a) and 3(b) for values of $\alpha=0.015$ and 0.05 , respectively. One readily checks that the tilted polar angle value needs to be much bigger than the actual tilt of $\hat{u}$ in order to reproduce the desired velocity. Moreover, a relationship between grain size and tilt of the easy axis is lacking. In the next section we focus on these two aspects, bringing into the discussion two features not considered by standard 1D models: internal DW dynamics and energy dissipation outside the wall.

\section{ENERGY DISSIPATION}

In the analytical model that we are considering, the system is allowed to dissipate energy only through the displacement and coherent precession of the domain wall, described by the two degrees of freedom $q(t)$ and $\phi(t)$. An additional degree of freedom can be introduced as variation of DW width $\Delta(t)$, but the results do not change considerably because the periodic widening and narrowing of the DW during the dynamics, the so called breathing, affects the instantaneous velocity $\dot{q}$ and at high fields and very short periods of precession this variation becomes negligible. On the other hand, an important consequence of internal DW dynamics is the emission of spin waves from the wall. This process transfers energy from the DW to the domains where it is quickly dissipated. Such mechanism, neglected by the 1D model of Walker and Slonczewski, has been investigated numerically in one-dimensional systems where it has been shown that spin waves emission from the DW can take place and contribute to DW motion when a strong hard-axis anisotropy is present in the system [29-31] and also in in-plane magnetized permalloy strips in presence of various forms of disorder [16,32]. In our system, both disorder and the fact that the wall is extended in length play a role, as discussed below. The extended DW 


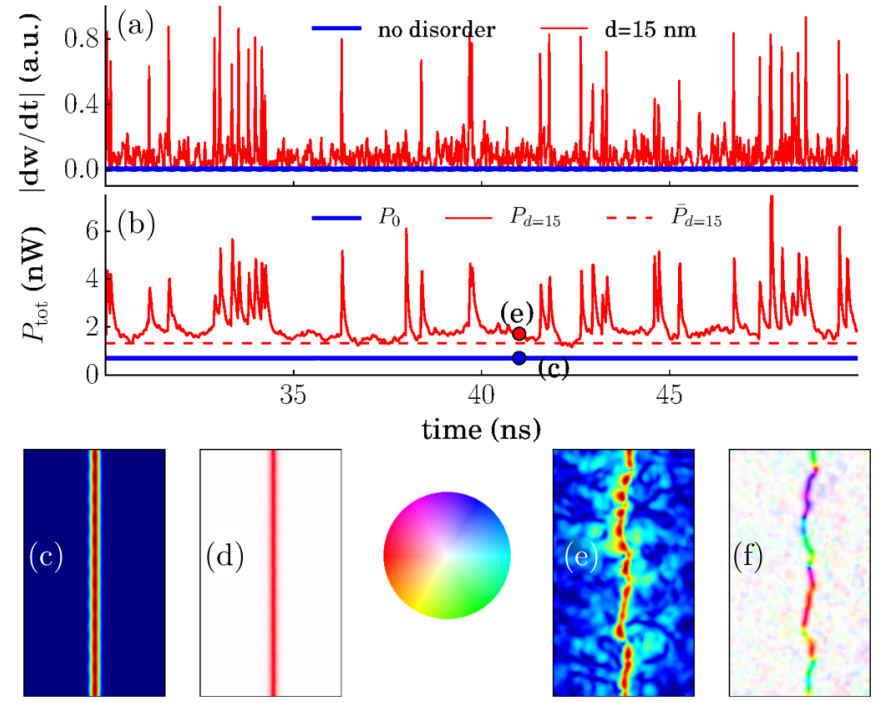

FIG. 4. (a) Time evolution of absolute topological charge variation calculated using Eq. (7) for a sample with no disorder (blue line) and another with grain size $d=15 \mathrm{~nm}$ (red line), extracted from simulations with $\alpha=0.015, \mu_{0} H=140 \mathrm{mT}$ and shown over a time window of $20 \mathrm{~ns}$. (b) Time evolution of the total dissipated power calculated using Eq. (2) for the same simulations as in (a), displayed over the same time window. It appears clear the correlation between peaks in dissipated energy and change in topological charge in the case with disorder. The dotted line marks the average dissipation rate $\bar{P}_{d}=\left\langle P_{d}-P_{0}\right\rangle$. (c) and (d) Snapshots of dissipated power and magnetization around the DW of the perfect sample at the instant of time indicated in (b) by the blue circle. (e) and (f) Snapshots showing the same features for the disordered sample at the instant of time indicated in (b) by the red circle. Hot-cold color gradient represents the amount of local dissipated power in logarithmic scale, in-plane magnetization angle is represented by a color as in the color wheel.

length allows for twisting of the magnetization along the DW. At high fields, this is not the case in a sample without disorder: as can be seen in the snapshot of magnetization in Fig. 4(d) the DW is uniformly magnetized and all the spins are precessing synchronously. This one-dimensional-like behavior is the reason for the good agreement of the analytical model with simulations without disorder. The introduction of disorder, however, yields a much more complex dynamics for the DW, as can be observed in the snapshot of Fig. 4(f), taken at the same instant and under the same applied field as in Fig. 4(d). Here the wall appears corrugated and the magnetization twists along the DW length, generating several VBLs: regions of the DW in which magnetization rotates in-plane, behaving as an in-plane wall inside the domain wall [28]. VBLs have a topological charge (or winding number) of $\pm 1 / 2$, depending on their chirality.

A recent study [11] presented the effect of interfacial Dzyaloshinsky-Moriya interaction (DMI) on DW dynamics in wide strips. DMI introduces an in-plane field acting on the wall, affecting the propagation of VBLs by giving different velocities to VBLs of different magnetic charge and causing them to annihilate in couples of same chirality and opposite magnetic charge. This feature is observed also in our simulations, where acceleration, deceleration, and annihilation of VBLs are caused by the different energy that VBLs have over grains with different in-plane anisotropy while propagating along a DW that is also able to curve its profile during dynamics [10,28]. In addition, the dynamics internal to the DW yields a much higher energy dissipation compared to that of a nondisordered sample: the in-plane anisotropy field, inhomogeneous along the DW length, creates a rich and varied landscape for the VBLs propagating inside the DW and for the DW in general. This results in oscillations and local breathing phenomena of the DW of complicated nature that, overall, generate a constant irradiation of spin waves from the DW. This constant additional dissipated power is absent in films with easy axis uniformly perpendicular to plane. Since VBLs have a topological charge, their complex dynamics in DWs propagating in disordered samples can be studied quantitatively by monitoring the time variation of the total topological charge $w$ of our sample, computed as [33]

$$
w(t)=\frac{1}{4 \pi} \int_{L} \int_{W} \mathbf{m}(t) \cdot\left(\frac{\partial \mathbf{m}(t)}{\partial x} \times \frac{\partial \mathbf{m}(t)}{\partial y}\right) d y d x .
$$

The absolute variation of topological charge is shown in Fig. 4(a) as the thick blue curve for a perfect sample and as the thin red curve for a disordered sample with grain size $15 \mathrm{~nm}$. As can be observed, the topological charge is conserved for DW dynamics in a perfect sample, while for samples with grains $\left|\frac{d w}{d t}\right|$ shows peaks corresponding to events of annihilation of couples of VBLs of the same topological charge. This is because VBLs are nucleated in couples of opposite topological charge $\pm 1 / 2$ that move in opposite direction, while annihilation takes place between VBLs of the same topological charge, so that a net change in $w$ of 1 unit is expected upon annihilation. Such particular dynamics has its origin in the different energy state of a VBL depending on its orientation and the in-plane orientation of the easy axis of the grain over which it is laying. This grain dependent in-plane anisotropy gives to VBLs grain dependent energy states and velocities, causing acceleration, deceleration, and even blocking of VBLs at grain boundaries where a sufficiently high energy barrier is found, generating annihilation events.

The thick blue line in Fig. 4(b) shows the power dissipation over time for the simulations on the perfect sample calculated using (2). As can be seen, a constant and low rate of energy dissipation is found, the reason being the 1D-like behavior of the system. The snapshot of Fig. 4(c), showing the spatial distribution of the dissipated energy, confirms that energy is being dissipated exclusively inside the DW and homogeneously distributed along its length, since the DW is the only region in which spins are precessing. On the other hand, in disordered samples more energy is dissipated during DW motion: the thin red curve in Fig. 4(b) shows the dissipated power in a disordered system with a DW propagating under the same applied field. The red curve describes a nonconstant dissipation of power, with peaks corresponding to VBL annihilation events, as confirmed by the agreement with the variation in the topological charge in Fig. 4(a). It is important to note the offset of the red curve, at about $1.5 \mathrm{nW}$, making clear the contribution that the complex dynamics internal to the DW, triggered by local nonzero in-plane anisotropy, has on energy dissipation. An additional check of this observation comes from the comparison of the distribution of energy dissipation 


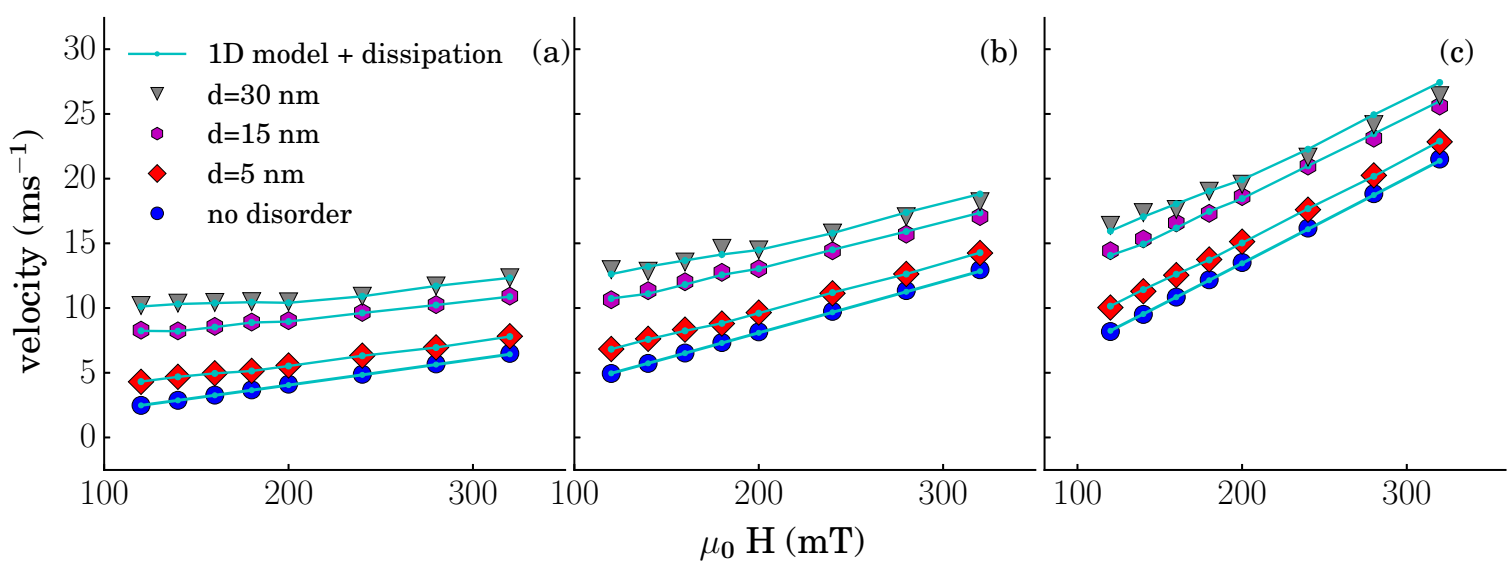

FIG. 5. (a) DW velocity versus applied field from simulations with $\alpha=0.015$ without disorder (blue circles) and with different grain sizes ( $d=5 \mathrm{~nm}$ red diamonds, $d=15 \mathrm{~nm}$ magenta hexagons, $d=30 \mathrm{~nm}$ gray down-triangles). Cyan dots show DW velocity computed from $1 \mathrm{D}$ model (11) with a realistic $\eta=4.5^{\circ}$ plus dissipation contribution extracted from simulations. Same results for $\alpha=0.03$ and $\alpha=0.05$ are shown in (b) and (c), respectively, where the dissipation contribution is the one extracted from the simulations in (a) with $\alpha=0.015$.

between a perfect and a disordered sample. Figure 4(e) shows that the dissipation of energy in disordered samples takes place not only inside the DW but also in the nearby domains, where spins are precessing due to spin wave emission even when no annihilation event is observed.

The fact that irradiation of energy from the DW to the domains and its subsequent faster dissipation is relevant in DW dynamics, appears also by looking at the variation of tilting angle $\eta$ required by the $1 \mathrm{D}$ model to fit the data when changing damping constant: $\alpha=0.015$ in Fig. 3(a) requires larger $\eta$ to fit simulations for $d=10 \mathrm{~nm}$ compared to $\alpha=0.05$ in Fig. 3(b), although the samples are exactly the same with identical distribution of $\hat{u}$ and $k_{u}$. Increasing Gilbert's damping, we are increasing the contribution of the applied field to DW velocity, reducing the effect of spin waves and making the 1D approximation more accurate at reproducing the micromagnetic simulation. In view of this, what can be done is to try to include in the $1 \mathrm{D}$ model the additional energy dissipated by the DW in those dynamics that cannot be described by a 1D model. Starting with conservation of energy from LLG equation (1):

$$
\frac{d \mathrm{E}}{d t}=-\frac{\alpha \mu_{0} M_{s}}{\gamma_{0}} \int_{V}\left(\frac{d \mathbf{m}}{d t}\right)^{2} d V,
$$

we can obtain its one-dimensional counterpart that takes into account easy-axis tilting:

$$
\begin{aligned}
-2 & \mu_{0} M_{s} H_{a} \dot{q}+2 \Delta\left(K \sin 2 \phi-2 k_{u} u_{1} u_{2} \cos 2 \phi\right) \dot{\phi} \\
= & \frac{-2 \alpha \mu_{0} M_{s}}{\gamma_{0}}\left[\frac{\dot{q}^{2}}{\Delta}+\Delta \dot{\phi}^{2}\right] .
\end{aligned}
$$

Within the 1D model framework, we are assuming that the only variation of internal energy comes from the displacement and the rotation of the DW spins and this is balanced by the work done by the viscous forces. Any dynamics taking place in the two domains of our system is neglected. To account for the energy dissipation that we observe in simulations with grains and tilted easy axis, we consider an average constant rate of energy dissipated into the domains rising from the complex dynamics of the incoherently precessing DW. To quantify it, we take the average dissipated power of our simulations with disorder, $\left\langle P_{d}\right\rangle$, corresponding to the average of the red line in Fig. 4(b), and we safely assume that in micromagnetic simulations of the perfect sample energy is dissipated exactly as prescribed by the $1 \mathrm{D}$ model. Then, the term that we need to add to the 1D model equation of conservation of energy is

$$
\bar{P}_{d}=\left\langle P_{d}\right\rangle-\left\langle P_{0}\right\rangle .
$$

This value, marked as the dashed red line in Fig. 4(b), represents the power dissipated by the system in other ways than simple precession of the spins inside the DW. It is dependent on $H_{a}$ since it relies on the dynamics triggered by the precession of the spins inside the DW and needs to be extracted from micromagnetic simulations for every applied field. Including this term in the energy conservation equation as done elsewhere [34], we have

$$
\begin{gathered}
-2 \mu_{0} M_{s} H_{a} \dot{q}+2 \Delta\left[K \sin (2 \phi)-2 k_{u} u_{1} u_{2} \cos 2 \phi\right] \dot{\phi} \\
=\frac{-2 \alpha \mu_{0} M_{s}}{\gamma_{0}}\left[\frac{\dot{q}^{2}}{\Delta}+\Delta \dot{\phi}^{2}\right]+\frac{\bar{P}}{t W} .
\end{gathered}
$$

Averaging this equation over a period of precession after using the expressions (4) and (5) for $\dot{q}^{2}$ and $\dot{\phi}^{2}$ we obtain

$$
\begin{aligned}
\langle v\rangle= & \frac{\gamma_{0} \Delta H_{W}}{1+\alpha^{2}}\left[\alpha h_{1}+\frac{1}{\alpha}\left(h_{1}-\sqrt{h_{1}^{2}-1-h_{2}^{2}}\right)\right] \\
& +\frac{\bar{P}}{2 \mu_{0} M_{s} H_{a} t W} .
\end{aligned}
$$

Extracting the value of $\bar{P}$ from micromagnetic simulations with a certain $\alpha$, the augmented model reproduces extremely well the DW velocity of the simulations also for other values of $\alpha$ as can be observed in Fig. 5, proving that the contribution to DW velocity introduced by disorder is the additional energy dissipated in the domains in the form of spin waves.

It remains to be explained the grain size dependence of this velocity shift. Since the distribution of $\hat{u}$ values is aleatory, the intensity and orientation of the in-plane field in neighboring grains is uncorrelated. DW motion takes place, as it has been shown, in an incoherent fashion. The nonuniform precession means precession via VBLs propagation, i.e., spins inside the 
DW precess by $\pi$ sweeps when a VBL passes past them. VBLs have a finite width $\Lambda=\sqrt{A / K}=28.9 \mathrm{~nm}$ which varies depending on the local orientation of $\hat{u}$. This means that the minimum size for coherent precession is $\sim 30 \mathrm{~nm}$ over which the $1 \mathrm{D}$ precessional motion with a uniform and tilted easy axis can be applied. When grain size is smaller than this value, the effective tilting of $\hat{u}$ is an average over $\Lambda$ of the different values over grains. Being the disorder uncorrelated, the local average results in a decrease of the in-plane component. This explains the saturation for $d$ close to $25-30 \mathrm{~nm}$. This argument also explains the lower dissipated energy at small $d$; VBLs propagate through an effectively more homogeneous medium and reduced effects of breathing and acceleration, deceleration with subsequent annihilation are observed [19].

\section{CONCLUSIONS}

In conclusion, we have used micromagnetic simulations to investigate domain wall motion in magnetic systems where a granular structure and interfacial effects are expected to play a role in introducing material inhomogeneities affecting DW dynamics. We showed that introducing disorder in simulations gives higher DW velocities in the high field regime while not considerably changing the mobility, and we identified the feature responsible for this velocity increase in the local variation of anisotropy easy-axis orientation. We addressed this problem first introducing an in-plane deviation of the anisotropy easy axis in the 1D model, proving that the feature was capable of qualitatively reproducing the shift. Second, we compared the dissipated power from micromagnetic simulations in a system with disorder with that of of a perfectly homogeneous system. We found that the local in-plane anisotropy field is the responsible for oscillations, local breathing phenomena of the DW and VBL annihilations that overall generate an irradiation of power from the DW to the domains which is constant over time and dependent on system characteristics such as the typical grain size and independent on the intrinsic Gilbert damping. This study evidences the fact that different models of disorder might have different effects on magnetization dynamics, stressing the importance of choosing the correct disorder models to reproduce experimental results.

\section{ACKNOWLEDGMENTS}

This work was supported by project WALL FP7-PEOPLE2013-ITN 608031 from the European Commission, project MAT2014-52477-C5-4-P from Spanish government, project SA282U14 and SA090U16 from Junta de Castilla y Leon.
The authors thank L. Herrera Diez and D. Ravelosona (Institut d'Électronique Fondamentale, Université Paris-Sud) for helpful discussion and comments.

\section{APPENDIX: ONE-DIMENSIONAL MODEL}

Here we present the 1D model and we derive all the expressions that are presented in the main text. To obtain Eq. (3), one has to proceed from LLG equations in polar coordinates and use an ansatz for a one-dimensional magnetic system with an up-down domain wall. Using the normalized form of the magnetization:

$$
\mathbf{M}(\mathbf{r}, t)=M_{s} \mathbf{m}(\mathbf{r}, t) .
$$

LLG equation (1) can be expressed in polar coordinates $\theta(\mathbf{r}, t), \varphi(\mathbf{r}, t)$ as

$$
\begin{gathered}
\dot{\theta}+\alpha \dot{\varphi} \sin \theta=-\frac{\gamma_{0}}{\mu_{0} M_{s} \sin \theta} \frac{\delta \mathcal{E}}{\delta \varphi}, \\
\dot{\varphi} \sin \theta-\alpha \dot{\theta}=\frac{\gamma_{0}}{\mu_{0} M_{s}} \frac{\delta \mathcal{E}}{\delta \theta},
\end{gathered}
$$

where $\mathbf{m}=\left(m_{x}, m_{y}, m_{z}\right)=(\sin \theta \cos \varphi, \sin \theta \sin \varphi, \cos \theta)$ and $-\frac{1}{M_{s} \sin \theta} \frac{\delta \mathcal{E}}{\delta \varphi},-\frac{1}{M_{s}} \frac{\delta \mathcal{E}}{\delta \theta}$ are the components of the effective field in polar coordinates.

Energy densities in polar coordinates are

$$
\begin{gathered}
\mathcal{E}_{\mathrm{ex}}=A\left[(\nabla \theta)^{2}+(\nabla \varphi)^{2} \sin ^{2} \theta\right], \\
\mathcal{E}_{\mathrm{an}}=k_{u}\left[1-(\mathbf{m} \cdot \hat{\mathbf{u}})^{2}\right], \\
\mathcal{E}_{\mathrm{dmg}}=\frac{\mu_{0} M_{s}^{2}}{2}\left[N_{z}+\sin ^{2} \theta\left(N_{x}-N_{z}\right)\right. \\
\left.+\sin ^{2} \theta \sin ^{2} \varphi\left(N_{y}-N_{x}\right)\right], \\
\mathcal{E}_{z}=-\mu_{0} M_{s} H_{a} \cos \theta,
\end{gathered}
$$

where we have assumed only a local contribution of the dipolar field. To account for the tilt of the easy axis we need to express the anisotropy energy term according to $\hat{u}=\left(u_{x}, u_{y}, u_{z}\right)=(\sin \eta \cos \zeta, \sin \eta \sin \zeta, \cos \eta)$ so that

$$
\begin{aligned}
\mathcal{E}_{\mathrm{an}}= & k_{u}\left[1-(\mathbf{m} \cdot \hat{\mathbf{u}})^{2}\right]=k_{u}\left(1-u_{z}^{2}\right)+k_{u} \sin ^{2} \theta\left(u_{z}^{2}-u_{x}^{2}\right) \\
& +k_{u} \sin ^{2} \theta\left[\sin ^{2} \varphi\left(u_{x}^{2}-u_{y}^{2}\right)-2 u_{x} u_{y} \sin \varphi \cos \varphi\right] \\
& -2 k_{u} \sin \theta \cos \theta\left(\cos \varphi u_{x}+\sin \varphi u_{y}\right) u_{z}
\end{aligned}
$$

and

$$
\begin{aligned}
\mathcal{E}_{\mathrm{an}}+\mathcal{E}_{\mathrm{dmg}}= & \underbrace{k_{u}\left(1-u_{z}^{2}\right)+\frac{\mu_{0} M_{s}{ }^{2}}{2} N_{z}}_{C_{\mathrm{st}}}+\sin ^{2} \theta \underbrace{\left[k_{u}\left(u_{z}^{2}-u_{x}^{2}\right)-\frac{\mu_{0} M_{s}{ }^{2}}{2}\left(N_{z}-N_{x}\right)\right]}_{K_{0}} \\
& +\sin ^{2} \theta[\sin ^{2} \varphi \underbrace{\left(k_{u}\left(u_{x}^{2}-u_{y}^{2}\right)-\frac{\mu_{0} M s}{2}\left(N_{x}-N_{y}\right)\right)}_{K}-2 k_{u} u_{x} u_{y} \sin \varphi \cos \varphi] \\
& -2 k_{u} \sin \theta \cos \theta\left(\cos \varphi u_{x}+\sin \varphi u_{y}\right) u_{z}
\end{aligned}
$$


is the full expression for the energy density of the so-called shape anisotropy.

We assume a magnetic sample of infinite length along the $x$ direction, with a rectangular cross section of finite thickness $t$ and width $W$. We assume our sample uniformly magnetized along thickness and width, while along its length magnetization varies following the conditions [see Fig. 1(a)]

$$
\begin{gathered}
\theta(x, t)=2 \arctan \left[\exp \left(\frac{x-q(t)}{\Delta}\right)\right], \\
\varphi(x, t)=\phi(t),
\end{gathered}
$$

describing an up-down domain wall configuration along the $x$ axis. In the above expressions $q(t)$ is the DW position along the $x$ axis, $\Delta$ is the DW width parameter, and $\phi(t)$ describes the in-plane orientation of the DW spins.

We obtain the relationships

$$
\begin{gathered}
\frac{\partial \theta}{\partial x}=\frac{\sin \theta}{\Delta}, \quad \frac{\partial \varphi}{\partial x}=0, \\
\delta \theta=-\frac{\sin \theta}{\Delta} d q, \quad \delta \varphi=d \phi, \\
\dot{\theta}=-\dot{q} \frac{\sin \theta}{\Delta}, \quad \dot{\varphi}=\dot{\phi} .
\end{gathered}
$$

Assuming this rigid magnetic configuration, $\mathcal{E}$ reduces to

$$
\begin{aligned}
\mathcal{E}= & A \frac{\sin ^{2} \theta}{\Delta^{2}}+\sin ^{2} \theta\left(K_{0}+K \sin ^{2} \phi-k_{u} u_{x} u_{y} \sin 2 \varphi\right) \\
& -2 k_{u} \sin \theta \cos \theta\left(\cos \varphi u_{x}+\sin \varphi u_{y}\right) u_{z}-\mu_{0} M_{s} H_{a} \cos \theta,
\end{aligned}
$$

where the constant energy term due to shape anisotropy, not affecting the dynamics, has been neglected.

In general, from LLG equations (A1) and (A2) we obtain

$$
\begin{gathered}
\delta \mathcal{E}=\frac{\delta \mathcal{E}}{\delta \theta} \delta \theta+\frac{\delta \mathcal{E}}{\delta \varphi} \delta \varphi \\
=-\frac{\mu_{0} M_{s}}{\gamma_{0}}[(\alpha \dot{\theta}-\dot{\varphi} \sin \theta) \delta \theta+(\dot{\theta}+\alpha \dot{\varphi} \sin \theta) \sin \theta \delta \varphi]
\end{gathered}
$$

and we eliminate the dependence on $\theta$ upon integration over $x$ :

$$
\begin{aligned}
d \sigma=\int_{x} \delta \mathcal{E} d x= & -\frac{\mu_{0} M_{s}}{\gamma_{0}}\left[\int_{-\infty}^{+\infty}(\alpha \dot{\theta}-\dot{\varphi} \sin \theta) \delta \theta d x\right. \\
& \left.+\int_{-\infty}^{+\infty}(\dot{\theta}+\alpha \dot{\varphi} \sin \theta) \sin \theta \delta \varphi d x\right]
\end{aligned}
$$

To do so, we make use of the expressions obtained with the rigid model (A9)-(A11) to have

$$
\begin{gathered}
d \sigma=-\frac{\mu_{0} M_{s}}{\gamma_{0}}\left[\left(\alpha \frac{\dot{q}}{\Delta}+\dot{\phi}\right) d q \int_{-\infty}^{+\infty} \frac{\sin ^{2} \theta}{\Delta} d x\right. \\
\left.+\left(-\frac{\dot{q}}{\Delta}+\alpha \dot{\phi}\right) d \phi \int_{-\infty}^{+\infty} \sin ^{2} \theta d x\right], \\
d \sigma=-\frac{2 \mu_{0} M_{s}}{\gamma_{0}}\left[\left(\alpha \frac{\dot{q}}{\Delta}+\dot{\phi}\right) d q+(-\dot{q}+\alpha \Delta \dot{\phi}) d \phi\right] .
\end{gathered}
$$

On the other hand, the linear wall energy $\sigma,\left(\mathrm{J} / \mathrm{m}^{2}\right)$ is simply

$$
\begin{aligned}
\sigma= & \int_{-\infty}^{+\infty} \mathcal{E} d x=\frac{2 A}{\Delta}+2 \Delta\left(K_{0}+K \sin ^{2} \phi\right. \\
& \left.-k_{u} u_{x} u_{y} \sin 2 \phi\right)-2 q \mu_{0} M_{s} H_{a},
\end{aligned}
$$

so that

$$
\begin{aligned}
d \sigma= & \frac{\partial \sigma}{\partial q} d q+\frac{\partial \sigma}{\partial \phi} d \phi=-2 \mu_{0} M_{s} H_{a} d q \\
& +2 \Delta\left(K \sin 2 \phi-2 k_{u} u_{x} u_{y} \cos 2 \phi\right) d \phi .
\end{aligned}
$$

Equating the differentials in the two expressions of $d \sigma$ [(A17) and (A19)] we obtain the two one-dimensional model equations:

$$
\begin{gathered}
\alpha \frac{\dot{q}}{\Delta}+\dot{\phi}=\gamma_{0} H_{a}, \\
\frac{\dot{q}}{\Delta}-\alpha \dot{\phi}=\frac{\gamma_{0}}{\mu_{0} M_{s}}\left(K \sin 2 \phi-2 k_{u} u_{x} u_{y} \cos 2 \phi\right) .
\end{gathered}
$$

It is useful to explicit the two expressions for $\dot{q}$ and $\dot{\phi}$,

$$
\begin{gathered}
\dot{q}=\frac{\gamma_{0} \Delta}{1+\alpha^{2}}\left(\alpha H_{a}+\frac{H_{K}}{2} \sin 2 \phi-H_{K}^{\prime \prime} \cos 2 \phi\right), \\
\dot{\phi}=\frac{\gamma_{0}}{1+\alpha^{2}}\left(H_{a}-\frac{\alpha H_{K}}{2} \sin 2 \phi+\alpha H_{K}^{\prime \prime} \cos 2 \phi\right),
\end{gathered}
$$

having introduced

$$
\begin{gathered}
H_{K}=\frac{2 K}{\mu_{0} M_{s}}, \\
H_{K}^{\prime \prime}=\frac{2 k_{u} u_{x} u_{y}}{\mu_{0} M s},
\end{gathered}
$$

where

$$
H_{W}=\frac{\alpha H_{K}}{2}, \quad h_{1}=\frac{H_{a}}{H_{W}}, \quad h_{2}=\frac{2 H_{K}^{\prime \prime}}{H_{K}} .
$$

Equation (A23) admits constant solutions when $h_{1}^{2} \leqslant 1+h_{2}^{2}$ or equivalently for

$$
H_{a} \leqslant \alpha \sqrt{\frac{H_{K}^{2}}{4}+H_{K}^{\prime \prime 2}} .
$$

We can proceed now to estimate the average velocity above Walker breakdown in the general case of a tilted easy axis. From

$$
\frac{d \phi}{d t}=\dot{\phi}=\frac{\gamma_{0} H_{W}}{1+\alpha^{2}}\left(h_{1}-\sin 2 \phi+h_{2} \cos 2 \phi\right)
$$

we have

$$
\frac{d \phi}{h_{1}-\sin 2 \phi+h_{2} \cos 2 \phi}=\frac{\gamma_{0} H_{W}}{1+\alpha^{2}} d t .
$$

Integrating over a period $T$ yields

$$
\frac{\gamma_{0} H_{W}}{1+\alpha^{2}} \int_{0}^{T} d T=\frac{\gamma_{0} H_{W}}{1+\alpha^{2}} T
$$


and

$$
\frac{\gamma_{0} H_{W}}{1+\alpha^{2}} T=\int_{0}^{2 \pi} \frac{d \phi}{h_{1}-\sin 2 \phi+h_{2} \cos 2 \phi}=\frac{2 \pi}{\sqrt{h_{1}^{2}-1-h_{2}^{2}}},
$$

so that the precessing period of the DW above Walker breakdown is

$$
T=\frac{2 \pi\left(1+\alpha^{2}\right)}{\gamma_{0} H_{W} \sqrt{h_{1}^{2}-1-h_{2}^{2}}} .
$$

The useful integrals for our calculations are

$$
\begin{gathered}
\frac{1}{T} \int_{0}^{T} \sin 2 \phi d t=\frac{h_{1}-\sqrt{h_{1}^{2}-1-h_{2}^{2}}}{1+h_{2}^{2}}, \\
\frac{1}{T} \int_{0}^{T} \cos 2 \phi d t=\frac{h_{2}\left(\sqrt{h_{1}^{2}-1-h_{2}^{2}}-h_{1}\right)}{1+h_{2}^{2}} .
\end{gathered}
$$

Using Eqs. (A22), (A28), and (A29) the average velocity over a period is

$$
\begin{aligned}
\langle v\rangle & =\langle\dot{q}\rangle_{T}=\frac{1}{T} \int_{0}^{T} \dot{q} d t \\
& =\frac{\gamma_{0} \Delta H_{W}}{1+\alpha^{2}}\left[\alpha h_{1}+\frac{1}{\alpha}\left(h_{1}-\sqrt{h_{1}^{2}-1-h_{2}^{2}}\right)\right] .
\end{aligned}
$$

[1] J. Akerman, Science 308, 508 (2005).

[2] I. Mihai Miron, G. Gaudin, S. Auffret, B. Rodmacq, A. Schuhl, S. Pizzini, J. Vogel, and P. Gambardella, Nat. Mater. 9, 230 (2010).

[3] S. Parkin and S.-H. Yang, Nat. Nanotechnol. 10, 195 (2015).

[4] Y. Zhang, W. S. Zhao, D. Ravelosona, J.-O. Klein, J. V. Kim, and C. Chappert, J. Appl. Phys. 111, 093925 (2012).

[5] K.-S. Ryu, L. Thomas, S.-H. Yang, and S. Parkin, Nat. Nanotechnol. 8, 527 (2013).

[6] N. L. Schryer and L. R. Walker, J. Appl. Phys. 45, 5406 (1974).

[7] A. P. Malozemoff and J. C. Slonczewski, Magnetic Domain Walls in Bubble Materials (Academic Press, New York, 1979).

[8] P. J. Metaxas, J. P. Jamet, A. Mougin, M. Cormier, J. Ferré, V. Baltz, B. Rodmacq, B. Dieny, and R. L. Stamps, Phys. Rev. Lett. 99, 217208 (2007).

[9] B. Hillebrands and A. Thiaville, eds., Domain-Wall Dynamics in Nanowiresand Nanostrips, Topics in Applied Physics Vol. 101 (Springer, Berlin, 2006).

[10] K. Yamada and Y. Nakatani, Appl. Phys. Express 8, 093004 (2015).

[11] Y. Yoshimura, K.-J. Kim, T. Taniguchi, T. Tono, K. Ueda, R. Hiramatsu, T. Moriyama, K. Yamada, Y. Nakatani, and T. Ono, Nat. Phys. 12, 157 (2015).

[12] L. Thevenard, C. Gourdon, S. Haghgoo, J.-P. Adam, H. J. von Bardeleben, A. Lemaître, W. Schoch, and A. Thiaville, Phys. Rev. B 83, 245211 (2011).

[13] C. Gourdon, L. Thevenard, S. Haghgoo, and A. Cēbers, Phys. Rev. B 88, 014428 (2013).

[14] S. Lemerle, J. Ferré, C. Chappert, V. Mathet, T. Giamarchi, and P. Le Doussal, Phys. Rev. Lett. 80, 849 (1998).

[15] P. Metaxas, in Solid State Physics-Advances in Research and Applications (Academic Press, New York, 2011), Vol. 62, pp. 75-162.
[16] H. Min, R. D. McMichael, M. J. Donahue, J. Miltat, and M. D. Stiles, Phys. Rev. Lett. 104, 217201 (2010).

[17] J. Leliaert, B. Van de Wiele, A. Vansteenkiste, L. Laurson, G. Durin, L. Dupré, and B. Van Waeyenberge, J. Appl. Phys. 115, 233903 (2014).

[18] T. Weindler, H. G. Bauer, R. Islinger, B. Boehm, J.-Y. Chauleau, and C. H. Back, Phys. Rev. Lett. 113, 237204 (2014).

[19] M. Voto, L. Lopez-Diaz, and L. Torres, J. Phys. D 49, 185001 (2016).

[20] C. Burrowes, N. Vernier, J.-P. Adam, L. Herrera Diez, K. Garcia, I. Barisic, G. Agnus, S. Eimer, J.-V. Kim, T. Devolder, A. Lamperti, R. Mantovan, B. Ockert, E. E. Fullerton, and D. Ravelosona, Appl. Phys. Lett. 103, 182401 (2013).

[21] K. Yamada, J.-P. Jamet, Y. Nakatani, A. Mougin, A. Thiaville, T. Ono, and J. Ferré, Appl. Phys. Express 4, 113001 (2011).

[22] L. Herrera Diez, F. García-Sánchez, J.-P. Adam, T. Devolder, S. Eimer, M. S. El Hadri, A. Lamperti, R. Mantovan, B. Ocker, and D. Ravelosona, Appl. Phys. Lett. 107, 032401 (2015).

[23] J.-P. Tetienne, T. Hingant, L. Martínez, S. Rohart, A. Thiaville, L. H. Diez, K. Garcia, J.-P. Adam, J.-V. Kim, J.-F. Roch, I. Miron, G. Gaudin, L. Vila, B. Ocker, D. Ravelosona, and V. Jacques, Nat. Commun. 6, 6733 (2015).

[24] R. Lo Conte, E. Martinez, A. Hrabec, A. Lamperti, T. Schulz, L. Nasi, L. Lazzarini, R. Mantovan, F. Maccherozzi, S. S. Dhesi, B. Ocker, C. H. Marrows, T. A. Moore, and M. Kläui, Phys. Rev. B 91, 014433 (2015).

[25] A. Vansteenkiste, J. Leliaert, M. Dvornik, M. Helsen, F. Garcia-Sanchez, and B. Van Waeyenberge, AIP Adv. 4, 107133 (2014).

[26] OOMMF User's Guide, Version 1.0 M.J. Donahue and D.G. Porter Interagency Report NISTIR 6376, National Institute of Standards and Technology, Gaithersburg, MD (Sep. 1999). 
[27] T. Devolder, P.-H. Ducrot, J. Adam, I. Barisic, N. Vernier, J.-V. Kim, B. Ockert, and D. Ravelosona, Appl. Phys. Lett. 102, 022407 (2013).

[28] T. Herranen and L. Laurson, Phys. Rev. B 92, 100405 (2015).

[29] R. Wieser, E. Y. Vedmedenko, and R. Wiesendanger, Phys. Rev. B 81, 024405 (2010).
[30] X. S. Wang, P. Yan, Y. H. Shen, G. E. W. Bauer, and X. R. Wang, Phys. Rev. Lett. 109, 167209 (2012).

[31] X. S. Wang and X. R. Wang, Phys. Rev. B 90, 184415 (2014).

[32] Y. Nakatani, A. Thiaville, and J. Miltat, Nat. Mater. 2, 521 (2003).

[33] H.-B. Braun, Adv. Phys. 61, 1 (2012).

[34] X. R. Wang, P. Yan, and J. Lu, Europhys. Lett. 86, 67001 (2009). 